\title{
Research on Influence of the Type of Cross Wind Field on the Aerodynamic Performance of a High-Speed Train
}

\author{
Y.H. Xi ${ }^{1}$, J. Mao ${ }^{1}$, Guowei Yang ${ }^{2}$, and Y. Fang ${ }^{1}$ \\ ${ }^{1}$ The Civil Institute of Beijing Jiaotong University, Beijing 100044, China \\ ${ }^{2}$ Institute of mechanics, Chinese academy of sciences, Beijing 100190, China
}

\begin{abstract}
Characteristic of cross wind field, such as uniform wind and lower atmospheric boundary layer wind, have directly influence on the operation safety of high-speed train which running in cross wind environment. In order to accurately assess its effect on the train running in flat land, flow field and aerodynamic characteristics of the high speed train running at $350 \mathrm{~km} / \mathrm{h}$ under different wind directions in these two types of Wind field mentioned above were simulated numerically and analyzed. The results show that, for a train running in the condition of the crosswind in flat land, aerodynamic lift, lateral force and overturning moment acting on the train increased rapidly with the wind direction angle increasing; when the train running through the lower atmospheric boundary layer, compared to an uniform wind filed, the pressure difference of the train at top, bottom and two sides were less, lateral force and overturning moment were also less, and aerodynamic lift had some uncertainty when the wind direction angle increased. Using the uniform wind for the operation safety evaluation of high-speed train which running in cross wind environment will overestimate risks the cross wind on the train operation safety, make the safety of the train too low to limit speed, thus affect the normal operation and the traffic efficiency. The lower atmospheric boundary layer wind was suggested to be used for the evaluation of the aerodynamic performance of a train.
\end{abstract}

Keywords: High-speed train, uniform wind, lower atmospheric boundary layer, numerical simulation, aerodynamic characteristics.

\section{Introduction}

Changes of aerodynamic characteristics of high-speed train in strong crosswind, especially the changes of aerodynamic lift, lateral force and overturning moment acting on the train, will affect the stability of running train, and the train is more likely to derail and overturn (Fujii et al. 1999; Anderssonl et al. 2004). Accident caused by strong wind happened occasionally around the world, which seriously threatened the passengers' life and wealth security (Orellano and Schoder 2003). Many numerical Simulations of aerodynamic characteristics of high-speed train in strong wind are calculated in various countries, which have proved the applicability of numerical simulation (Carrarini 2007; Li et al. 2009), and the safety problems caused by crosswind effect are deeply analyzed (Xi et al. 2010; Zen et al. 2006). 
The crosswind fields are uniform in the majority of these simulations and analyses. But it does not correspond to the realistic exponential distribution regularity of wind field velocity, so it's against to estimating the safety of high-speed train under crosswind accurately. Lately, some researchers use natural wind field, namely lower atmospheric boundary layer wind,on study of aerodynamic load acting on static train and container train on bridge (Gao and Miao 2010; Jiang et al. 2010). There's no paper presented aerodynamic load acting on high-speed train with more than $300 \mathrm{~km} / \mathrm{h}$ using lower atmospheric boundary layer wind. As the high-speed train runs rapidly, and the stability relates to crosswind closely, it is required to calculate aerodynamic forces caused by side wind,in order to provide basis for analyzing track dynamic characteristics accurately,estimating how side wind impact high-speed train, and determine speed-limit control of high-speed train under strong wind. The article takes a high-speed train running on plain for example, modelling based on the authentic appearance and detailed structure, employing uniform wind at the same standard wind velocity and lower atmospheric boundary layer wind, computes and analyzes external flow field and aerodynamic forces of high-speed train moving at the rate of $350 \mathrm{~km} / \mathrm{h}$ under different side winds.

\section{Calculation Model and Method}

According to a real high-speed train on operation in our country, crosswind effect of a plain-running train is analyzed using numerical simulation as below.

\subsection{Computational Model and Conditions}

The middle part cross section of a train is invariant, and a decrease in length of train model does not make essential characteristics different (Khier et al. 2000). So, as a simplification, the length of the train was limited to that of a train assembly consisting of three trains, head train, intermediate train and tail train, the length of the trains are $26 \mathrm{~m}, 25 \mathrm{~m}, 26 \mathrm{~m}$, and the height is $3.9 \mathrm{~m}$, considering detailed structure like bogies and pantograph. The head and tail trains are of streamline shape. The main calculation and working conditions are:

(1) The train is running at $350 \mathrm{~km} / \mathrm{h}(97.22 \mathrm{~m} / \mathrm{s})$; using uniform wind and lower atmospheric boundary layer wind as side wind respectively, of which the velocity is $25 \mathrm{~m} / \mathrm{s}$ at the meteorological height of $10 \mathrm{~m}$; the angles of side wind are $30^{\circ}, 60^{\circ}$ and $90^{\circ}$ separately.

(2) The train is running at $350 \mathrm{~km} / \mathrm{h}$ with the influence of a crosswind at $25 \mathrm{~m} / \mathrm{s}$, and resultant velocity is close to or more than $1 / 3$ velocity of sound under different side wind directions. So the flow is regarded three-dimensional, viscous and compressible. Governing equations are determined by the reference (Wang 2004).

(3) Simulate external flow field around train under relative motion condition. Set the train static, and the floor moving. The floor has the velocity equal to that of the train speed and is opposite to its direction. The wind relative to a vehicle is the resultant of a natural wind vector and the wind induced by train running, as shown in Figure 1.

Where, $v_{\text {inlet } 1}=V+W \cos \beta ; v_{\text {inlet } 2}=W \sin \beta$. Unit of velocity is $\mathrm{m} / \mathrm{s}$. 


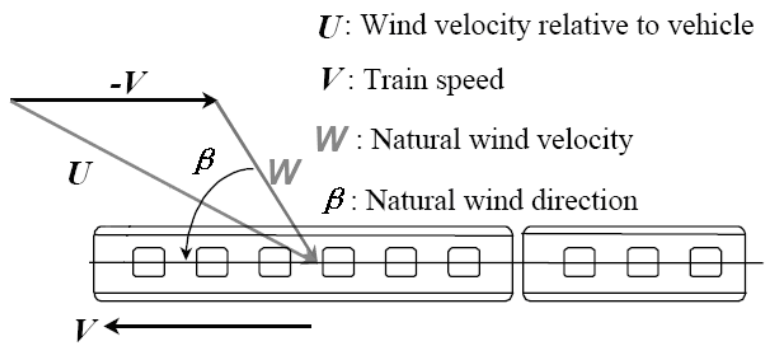

Fig. 1 Natural wind velocity and wind velocity relative to vehicle

\subsection{Computational Domain and Mesh Design}

The size of the computational domain was consistent with that used by Okumura and Kuriyama (Krajnovic and Siniša 2009), see Figure 2. Use trim grids, which is similar to numerical simulation of model test. And use wall function method on the surface of train and the floor. Number of wall boundary layers is initially set to be 6 , totally $4.86 \mathrm{~mm}$, the distance between the first layer grids and the wall is $0.3 \mathrm{~mm}$. And Number of floor boundary layers is initially set to be 6 , totally $4.86 \mathrm{~mm}$, the distance between the first layer grids and the wall is $0.3 \mathrm{~mm}$. The total number of mesh cells in the computational domain for the model was approximately $3 \times 10^{7}$. The optimized number of both wall and floor boundary layers is 3 , the thickness is $2.925 \mathrm{~mm}$, and the distance between the first layer grids and the wall is $0.3 \mathrm{~mm}$. It's of high-density where great changes take place in the flow field, like wake flow, train surface, pantograph, etc, see figure 3 . The total number of mesh cells in the computational domain for the model is about $1.7 \times 10^{7}$. The minimum grid size of refined and unrefined regions is $0.1 \mathrm{~mm}$ and $0.2 \mathrm{~mm}$, respectively.

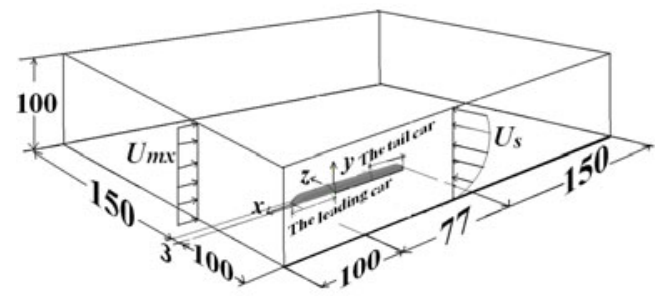

Fig. 2 Computational domain

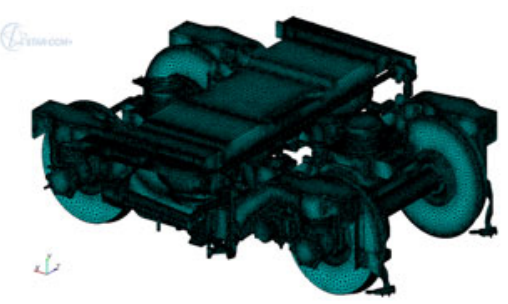

Fig. 3 Grid system 


\subsection{Boundary Conditions}

The boundary conditions used in the calculations are as follows:

(1) Inlet of the region. Use this velocity boundary conditions: when the crosswind is uniform, $v_{w}=25 \mathrm{~m} / \mathrm{s}$; and when it's lower atmospheric boundary layer wind, $v_{w}$ is determined according to the exponential distribution regularity in height direction (along y-axis): $v_{w y} / v_{w y 0}=\left(y / y_{0}\right)^{0.16}$, where $v_{w y}=v_{w}, y_{0}=10$ $\mathrm{m} ; v_{w \mathrm{y} 0}$ is the side wind velocity when $\mathrm{y}=10 \mathrm{~m}$, and $v_{w \mathrm{y} 0}=25 \mathrm{~m} / \mathrm{s}$.

(2) Outlet of the region. Use constant pressure condition, and the outlet pressure is one standard atmosphere;

(3) Train surface. Boundary-layer effection impacts on train surface, so the train surface is treated as slip boundary.

(4) The floor. Use move-floor method to eliminate the influence from floor effect. The speed of moving floor equals to the speed of inlet velocity, $v_{g}=v_{m x}=v_{t}+v_{w} \cos \beta$;

(5) Upper surface of computational domain. The computational domain of the flow is big enough, so the geometry boundaries of calculation region have little impact on flow field around the train. The upper surface is treated as noslip boundary.

\section{Results and Discussion}

In order to investigate the impact that uniform wind and lower atmospheric boundary layer wind have on aerodynamic performance of high-speed train, pressure distribution of around the train and on the surface of the train, along with eddy transformation close-by the train, are discussed.

\subsection{Comparisons of Pressure Field and Streamline}

\subsubsection{Influence of Characteristics of Cross Wind Field on Pressure Field and Streamline}

Figure 4 and Figure 5 show the pressure field and streamline in a typical condition, on the cross section of the train where $x=-38 m$, (located on tail train, $12.85 \mathrm{~m}$ away from nose cone of tail train). The figures indicate that different cross wind fields make different pressure fields and streamlines, and the differences are obvious in some cases.

On the aspect of pressure field, the positive-pressure area and pressure value of windward surface of the train in lower atmospheric boundary layer wind condition is obviously less than that in uniform wind condition, and the position of positive pressure is higher. The negative pressure area of leeward surface of the train in lower atmospheric boundary layer wind condition is obviously less than that in uniform wind condition, while the pressure value is higher. The figures show that 
vertex angles $\mathrm{A}_{1}, \mathrm{~B}_{1}, \mathrm{C}_{1}$ and $\mathrm{A}_{1}{ }^{\prime}, \mathrm{B}_{1}{ }^{\prime}, \mathrm{C}_{1}{ }^{\prime}$ at upper windward side, as well as some other region present negative pressure apparently, and the pressures of lower atmospheric boundary layer wind are higher than that of uniform wind; vertex angles $\mathrm{A}_{2}, \mathrm{~B}_{2}, \mathrm{C}_{2}$ and $\mathrm{A}_{2}{ }^{\prime}, \mathrm{B}_{2}{ }^{\prime}, \mathrm{C}_{2}{ }^{\prime}$ at lower windward side, as well as some other regions also present negative pressure, but the pressures are close to atmosphere.

On the aspect of flow field, the split-flow points on windward side locate differently, the split-flow point of lower atmospheric boundary layer wind is at a higher location. Two dissimilar size vortexes emerge from both of the two different winds, but the sizes and locations differ. The flow around train of uniform wind is more prone to breakaway from the top and the bottom of the train than that of lower atmospheric boundary layer wind, the size of vortexes emerge from uniform wind are larger, and impact on the train body at a longer distance, these indicate that uniform wind is affected more than lower atmospheric boundary layer wind on downstream flow of flowing around train.

\subsubsection{Influence of Crosswind Angles on Pressure Field and Streamline}

Figure 4 shows that relative pressure distributions of the windward side, leeward side, top and bottom of train change apparently as the crosswind angles vary with couple of side wind and train wind in these two wind field. The bigger side wind angle, the larger windward pressure and the smaller leeward pressure, and the negative pressure area on windward vertex angle is more obvious. The changes of lower atmospheric boundary layer wind are not as remarkable as that of uniform wind. The pressure field differs more when the side wind angle is large.

\subsubsection{Influence of Crosswind Angles on Train-Surface Pressure}

The pressure fields differ along with different side wind fields, thus making the aerodynamic loads different. Fig 5 presents contrast curves of train-surface pressure on a cross section at tail train $\mathrm{x}=-38 \mathrm{~m}$ when crosswind angle is $90^{\circ}$, pressure reaches the maximum at the split-flow on the windward of train, and the maximum positive pressure of uniform wind is $182.33 \mathrm{~Pa}$ larger than that of lower atmospheric boundary layer wind. The pressure of uniform wind is the same with the pressure of lower atmospheric boundary layer wind, is negative, on a point of transition region between windward side and the top of the train when it is about $3.25 \mathrm{~m}$ to the floor (the height of the train is $3.9 \mathrm{~m}$ ) at various wind angles. The pressure becomes smaller when the position is higher, and the pressure of lower atmospheric boundary layer wind is larger than that of uniform wind. On the leeward side of the train, surface pressures are all negative, and that of the uniform wind is smaller. The maximum pressure difference is $70.79 \mathrm{~Pa}$ at the same position, and the position in height of the minimum pressure varies with side wind angle. The surface pressure of both uniform wind and lower atmospheric boundary layer wind is negative on the top and bottom of the train, and it is the same in variation trend with position and crosswind angle. The pressure distribution on the top is not asymmetry, the minimum pressure is $0.8 \mathrm{~m}$ to midline at windward side; The pressure of lower atmospheric boundary layer wind is higher than the pressure of uniform wind in various angles, the maximum difference is $350.41 \mathrm{~Pa}$; the difference goes smaller when it is close to leeward side. While the pressure 
distribution on the bottom that is close to leeward side is relatively uniform, and the pressure of lower atmospheric boundary layer wind is higher than the pressure of uniform wind in various angles, the maximum difference is $118.41 \mathrm{~Pa}$. There's a similar variation trend with surface pressure on constant section of intermediate train and head train and that of tail train.

It is clear that, to compare lower atmospheric boundary layer wind with uniform wind, the amplitude of variation of windward pressure is obviously bigger than that of the leeward, while the amplitude of variation of base pressure is obviously smaller than that of the top pressure. To use uniform wind will overrate adverse effect that surface pressure distribution act on aerodynamic force of train.

The reason for that difference is the distinct of velocity boundary conditions when using uniform wind and lower atmospheric boundary layer wind on numerical calculation. The lower atmospheric boundary layer wind considers the
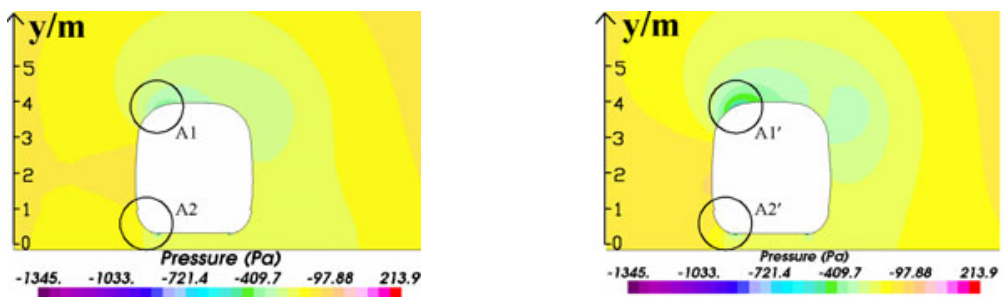

(a) Lower atmospheric boundary layer wind, (a') Uniform wind, crosswind angle $30^{\circ}$ crosswind angle $30^{\circ}$
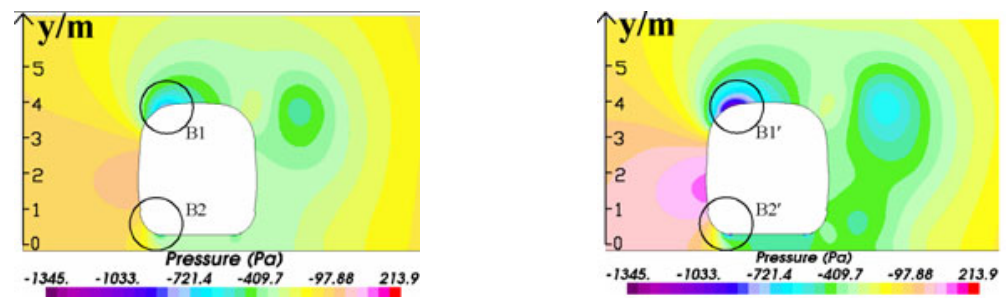

(b) Lower atmospheric boundary layer wind, (b') Uniform wind, crosswind angle $60^{\circ}$ crosswind angle $60^{\circ}$
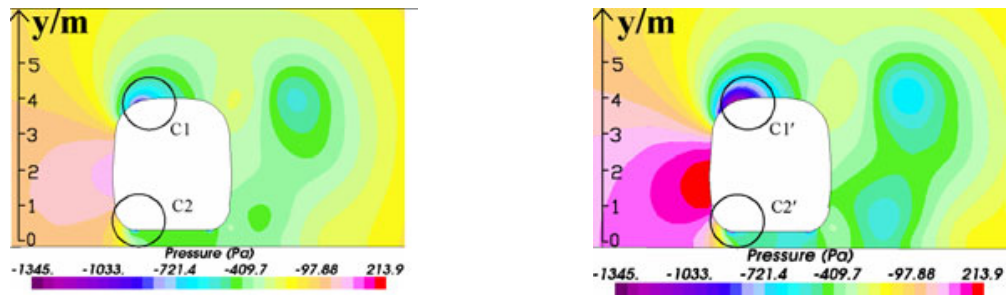

(a) Lower atmospheric boundary layer wind, (c') Uniform wind, crosswind angle $90^{\circ}$ crosswind angle $90^{\circ}$

Fig. 4 Distribution of the relative pressure around the train 
impact on velocity distribution from air viscous shearing stress, which reflect the fundamental characteristics of real flow field; and the uniform wind doesn't take velocity gradient into consideration, it is only applied to wind tunnel testing environment. The different velocity boundary conditions bring different flow field around train, and the air flow in crosswind of uniform wind is more than that of lower atmospheric boundary layer wind, thus leading to a different pressure distribution of train surface.

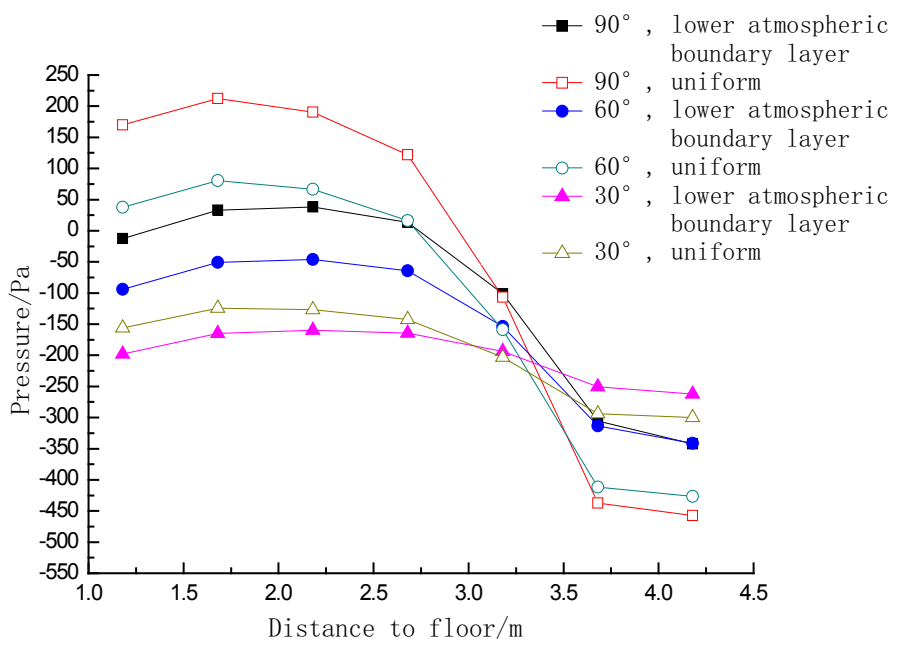

(a) Tail train, windward (the distance to floor corresponding to Figure 4 )

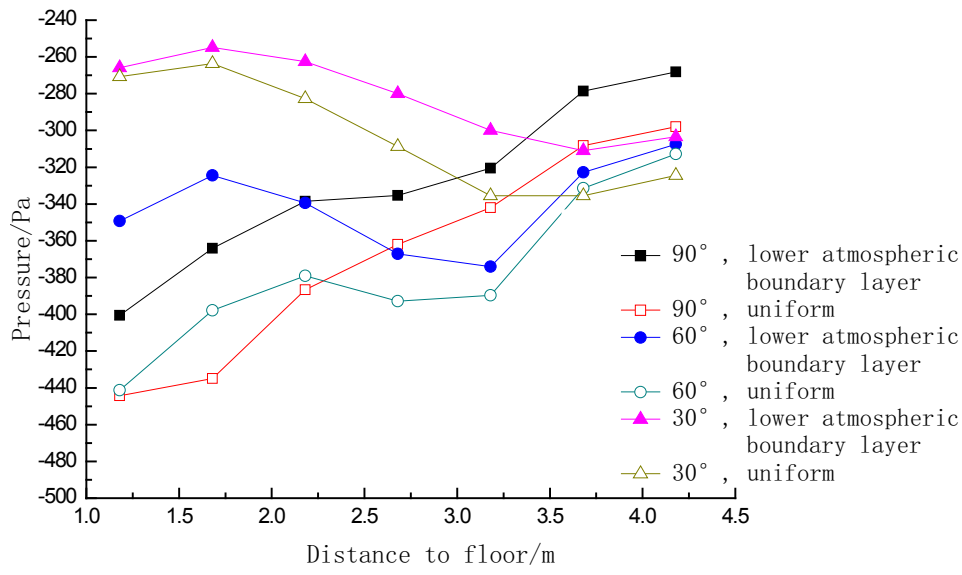

(b) Tail train, leeward (the distance to floor corresponding to Figure 4)

Fig 5 Changes of the relative pressure on the surface of the train 


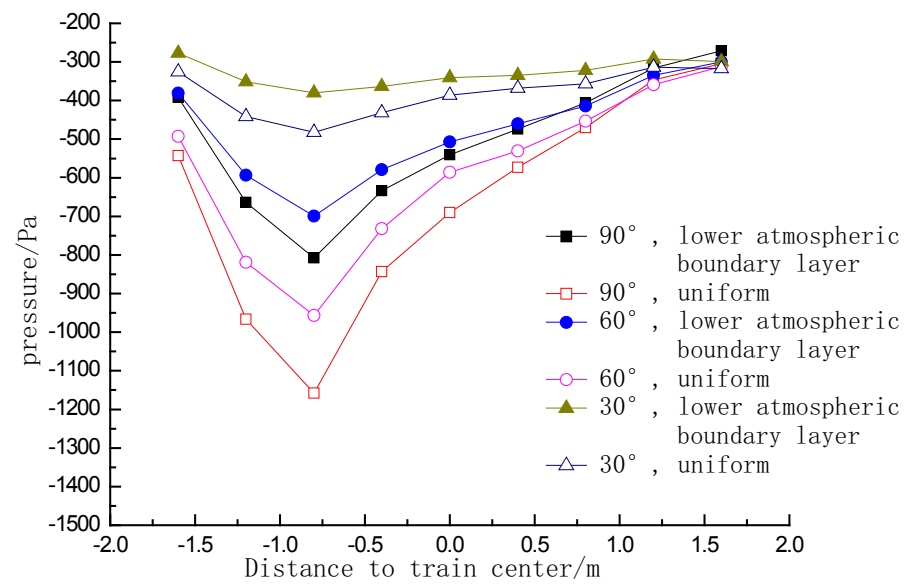

(c) Tail train, top (the distance to floor corresponding to Figure 4)

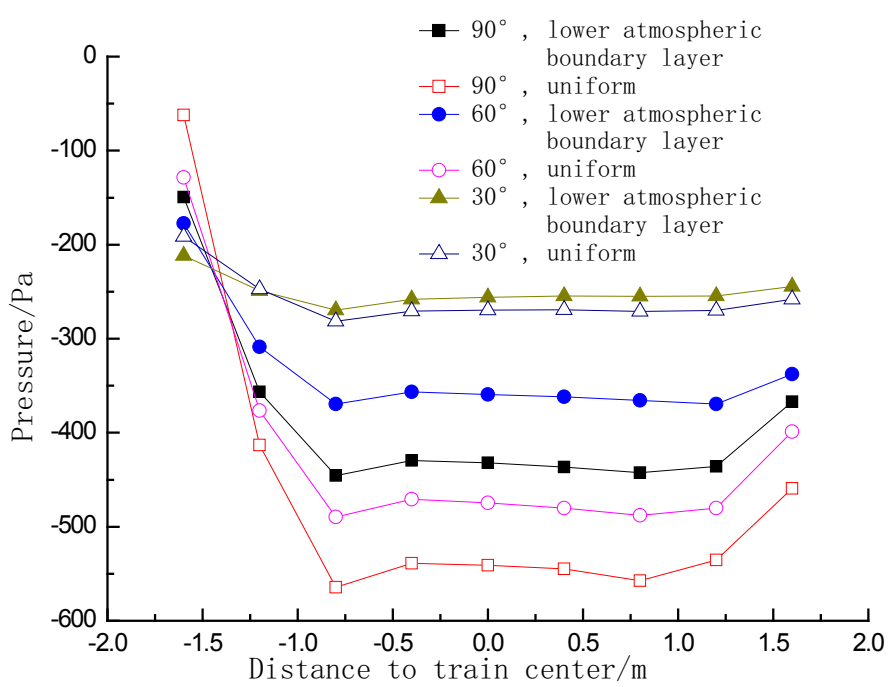

(d) Tail train, bottom (the distance to floor corresponding to Figure 4)

Fig. 5 (continued)

\subsection{Performance Comparison on Aerodynamic Force}

It's realized from the result that there are some differences on pressure distribution and flow field between uniform wind and lower atmospheric boundary layer wind. So these two cross wind fields create different aerodynamic forces on train. The aerodynamic forces impact running safety under crosswind conditions directly, lift $F_{y}$, lateral force $F_{z}$ and overturning moment $M_{x}$ are of the most influential quantities on the safety of a running train. 
The overturning moment is defined as a product of aerodynamic force and the distance to leeward top track, positive clockwise, it's the sum of moments produced by lift and lateral force. Figure 6 shows the comparison of lift, lateral force and overturning moment acting on high-speed train in conditions of uniform wind and lower atmospheric boundary layer wind.

Figure 6 indicates that in both uniform wind and lower atmospheric boundary layer wind, lift, lateral force and overturning moment acting on train are bigger if the side wind angle is big, and the head train, intermediate train and tail train contribute differently to the whole-train force. The forces go bigger along with the side wind angles, but the ranges are different quantitatively, the head train is the most sensitive to the angle and the tail train is the least. Meanwhile the aerodynamic-force difference is obvious of uniform wind and lower atmospheric boundary layer wind.

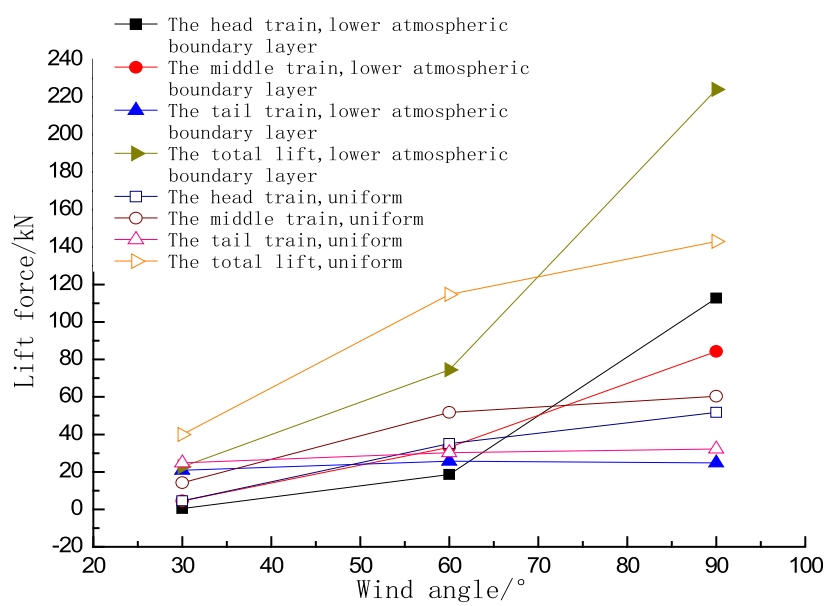

(a) Lift force versus the wind angle

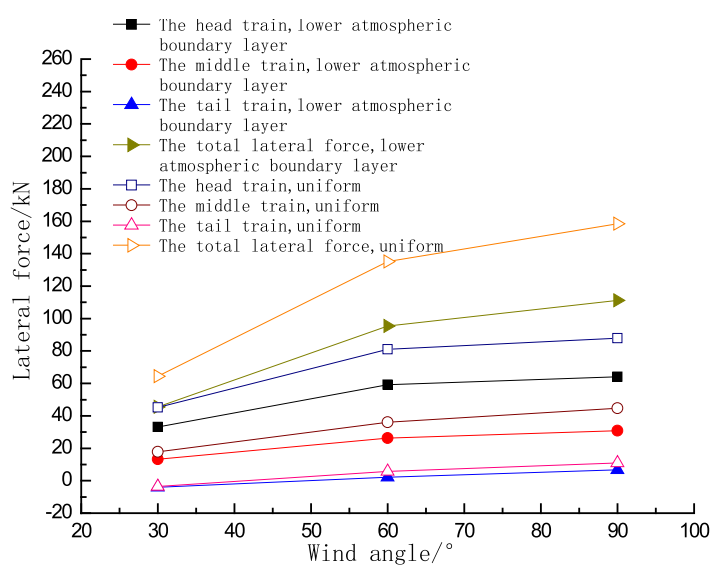

(b) Lateral force versus the wind angle

Fig. 6 Relationship between the aerodynamic forces and the angle of the cross wind 


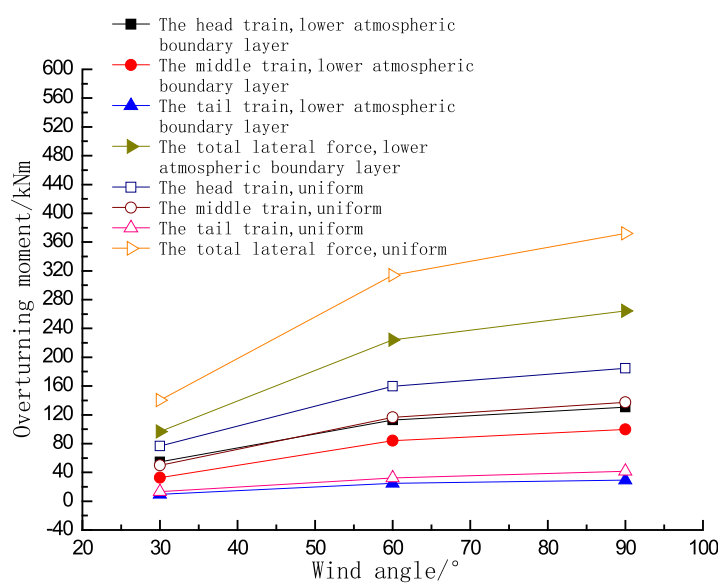

(c) Overturning moment versus the wind angle

Fig. 6 (continued)

It can be seen from Figure 4 and Figure 5 that the pressure of the upper and lower surfaces is negative, and the upper-surface pressure is much lower, and it's integrated into upward different pressure lift. Figure 6(a) shows that ,in lower atmospheric boundary layer wind, lift on tail train is the largest when the side wind angle is less than $60^{\circ}$; lift on intermediate train becomes the largest when the side wind angle is $60^{\circ}$; lift on head train goes the largest when the side wind angle is less than $90^{\circ}$. While in uniform wind, lift on tail train is the largest when the side wind angle is less than $60^{\circ}$; lift on intermediate train becomes the largest when the side wind angle is more than $60^{\circ}$. If side wind angle is no more than $60^{\circ}$, the total lift in lower atmospheric boundary layer wind is similar to that in uniform wind, with the maximum difference of $40.18 \mathrm{kN}$; if side wind angle is about $90^{\circ}$, the air velocity of train bottom is lower than that of the top in the condition of lower atmospheric boundary layer wind, which is more obvious than the phenomenon in uniform wind, so the total lift in lower atmospheric boundary layer wind is larger than that in uniform wind.

Figure 4 and Figure 5 also show that with the augment of side wind angle, the vortex emerges from crosswind more significant, the proportion of energy consumption caused by vortex increases, the pressure difference of two sides enlarges, and the lateral forces increase as well. Figure 6(b) indicates that for both lower atmospheric boundary layer wind and uniform wind, the aerodynamic lateral force acting on a partial or a running whole train increases with the increase of side wind angle, and the value reaches the maximum at the side wind angle of $90^{\circ}$. When the angle is less than $90^{\circ}$, the maximum lift is on the head train, the minimum on the tail train, and the middle on the intermediate train. The lateral forces are smaller in lower atmospheric boundary layer wind than that in uniform wind, for head train, middle train, tail train or complete train. And the difference can reach $47.27 \mathrm{kN}$ at most. 
Overturning moment is a main factor for derailment and overturning. Figure 6(c) shows that for both lower atmospheric boundary layer wind and uniform wind, the overturning moment acting on a partial or a running whole train increases with the increase of side wind angle, and the value reaches the maximum at the wind angle $90^{\circ}$. The larger wind angle is, the more dangerous for derailment and overturning. When the angle is less than $90^{\circ}$, the maximum overturning moment is on the head train, and the head train is the most prone to derailment and overturning; the minimum on the tail train and it is relatively safe; the middle overturning moment and safety on the intermediate train. The overturning moment are smaller in lower atmospheric boundary layer wind than that in uniform wind, for head train, middle train, tail train or whole train. And the difference can be $107.61 \mathrm{kN} \cdot \mathrm{m}$ at most.

Table 1 Comparison of computational aerodynamic force

\begin{tabular}{|c|c|c|c|c|c|c|c|c|}
\hline Wind angle & Part & Fyj $(\mathrm{kN})$ & Fye $(\mathrm{kN})$ & $\begin{array}{l}\Delta \mathrm{Fy} \\
\text { Fye }\end{array}$ & \begin{tabular}{|l|}
$\Delta \mathrm{Fz}$ \\
$(\mathrm{kN})$
\end{tabular} & $\begin{array}{l}\Delta \mathrm{Fz} \\
\mathrm{Fze}\end{array}$ & $\Delta \mathrm{Mx}(\mathrm{kN} \cdot \mathrm{m})$ & $\begin{array}{l}\Delta \mathrm{Mx} \\
\mathrm{Mxe}\end{array}$ \\
\hline \multirow{4}{*}{$30^{\circ}$} & $\begin{array}{l}\text { Head } \\
\text { train }\end{array}$ & 4.47 & 0.47 & $848.47 \%$ & 12.01 & $36.25 \%$ & 21.87 & $39.93 \%$ \\
\hline & $\begin{array}{c}\text { Intermediate } \\
\text { train }\end{array}$ & 14.22 & 4.59 & $209.77 \%$ & 4.52 & $34.05 \%$ & 17.28 & $52.9 \%$ \\
\hline & $\begin{array}{l}\text { Tail } \\
\text { train }\end{array}$ & 24.77 & 20.79 & $19.11 \%$ & 0.47 & $11.41 \%$ & 3.74 & $38.94 \%$ \\
\hline & $\begin{array}{c}\text { Complete } \\
\text { train }\end{array}$ & 39.90 & 22.66 & $76.08 \%$ & 18.94 & $41.59 \%$ & 43.42 & $44.9 \%$ \\
\hline \multirow{4}{*}{$60^{\circ}$} & $\begin{array}{c}\text { Head } \\
\text { train }\end{array}$ & 35.02 & 18.63 & $88 \%$ & 21.75 & $36.73 \%$ & 46.77 & $41.46 \%$ \\
\hline & $\begin{array}{c}\text { Intermediate } \\
\text { train }\end{array}$ & 51.81 & 33.19 & $56.07 \%$ & 9.77 & $37.18 \%$ & 32.30 & $38.36 \%$ \\
\hline & $\begin{array}{l}\text { Tail } \\
\text { train }\end{array}$ & 30.21 & 25.74 & $17.34 \%$ & 3.63 & $173.01 \%$ & 7.30 & $29.42 \%$ \\
\hline & $\begin{array}{c}\text { Complete } \\
\text { train }\end{array}$ & 114.66 & 74.48 & $53.96 \%$ & 39.87 & $41.83 \%$ & 89.79 & $40.06 \%$ \\
\hline \multirow{4}{*}{$90^{\circ}$} & $\begin{array}{c}\text { Head } \\
\text { train }\end{array}$ & 51.70 & 112.79 & $54.16 \%$ & 23.78 & $37.08 \%$ & 53.89 & $41.26 \%$ \\
\hline & $\begin{array}{c}\text { Intermediate } \\
\text { train } \\
\end{array}$ & 60.37 & 84.20 & $28.31 \%$ & 13.75 & $44.48 \%$ & 37.68 & $37.82 \%$ \\
\hline & $\begin{array}{l}\text { Tail } \\
\text { train }\end{array}$ & 32.19 & 24.80 & $29.81 \%$ & 4.31 & $64.57 \%$ & 12.29 & $41.94 \%$ \\
\hline & $\begin{array}{c}\text { Complete } \\
\text { train }\end{array}$ & 142.98 & 224.15 & $36.21 \%$ & 47.27 & $42.5 \%$ & 107.61 & $40.73 \%$ \\
\hline
\end{tabular}

Table 1 presents the differences on aerodynamic forces between lower atmospheric boundary layer wind and uniform wind. Fyj is lift force on train in uniform wind; $\mathrm{F}_{y e}, \mathrm{~F}_{\mathrm{ze}}$ and $\mathrm{M}_{\mathrm{x} e}$ are lift, lateral force and overturning moment in lower atmospheric boundary layer wind, respectively; $\Delta \mathrm{F}_{\mathrm{y}}, \Delta \mathrm{F}_{\mathrm{z}}$ and $\Delta \mathrm{M}_{\mathrm{x}}$ are the 
differences of lift, lateral force and overturning moment between uniform wind and lower atmospheric boundary layer wind and uniform wind. It can be seen from the table that the aerodynamic forces and overturning moments acting on a partial or a whole running train are higher in uniform wind. Comparing with those in lower atmospheric boundary layer wind, which is more approximate to the reality, the higher-part percentages for lift, lateral force and overturning moment of the complete train are as high as 76.08, 41.59, 44.9 (side wind angle $30^{\circ}$ ); 53.96, 41.83, 40.06(side wind angle 60); 36.21, 42.5, 40.73 (side wind angle $90^{\circ}$ ). Estimating the crosswind safety of train according to this will overrate the unsafety of running train in crosswind.

\section{Conclusions}

In the view of the results of the presented numerical simulation, the following conclusions on estimating aerodynamic characteristics of high-speed train running on plain in crosswind condition are drawn.

(1) The lift force of whole train increases with the increase of wind angle for both lower atmospheric boundary layer wind and uniform wind. The total lift force is larger in uniform wind when the wind angle is less than $60^{\circ}$, and the total lift force is larger in lower atmospheric boundary layer wind when the wind angle is about $90^{\circ}$.

(2) The lateral force of partial and whole train increases with the increase of wind angle for both lower atmospheric boundary layer wind and uniform wind. The lateral forces are smaller in lower atmospheric boundary layer wind than that in uniform wind, for head train, middle train, tail train and complete train.

(3) The overturning moment acting on a partial or a complete running train increases with the increase of side wind angle for both lower atmospheric boundary layer wind and uniform wind, and the dangerous for derailment and overturning increases as well; When the crosswind angle is less than $90^{\circ}$, the maximum overturning moment is on the head train, so the head train is the most prone to derailment and overturning; the overturning moments are smaller in lower atmospheric boundary layer wind than that in uniform wind, for head train, middle train, tail train and complete train.

(4) Estimating the crosswind safety of train according to the result calculated in uniform wind will overrate the unsafety of running train in crosswind and the safety running speed of train will be restrict lower, thus influencing the normal operation and working efficiency of the train. Estimating the safety according to the result calculated in lower atmospheric boundary layer wind which is more approximate to the reality is suggested.

Acknowledgments. The authors wish to acknowledge the support and motivation provided by 11th Five year plan of China in Ministry of Railways "Research of Key Techniques and Development of Key Equipments of High-Speed Train"(No. GTYZ200902). 


\section{References}

Fujii, T., Maeda, T., Ishida, H.: Wind-induced Accidents of Train/Vehicles and Their Measure in Japan. Quarterly Report of Railway Technical Research Institute 1, 50-55 (1999)

Anderssonl, E., Haggstrom, J., Sima, M.: Assessment of train-overturning risk due to strong cross-winds. J. Rail and Rapid Transit, Proc. Instn. Mech. Engrs. 218(F), 213-223 (2004)

Orellano, A., Schoder, M.: On side-wind stability of high speed trains. Vehicle System Dynamics Supplement 40, 143-160 (2003)

Carrarini, A.: Reliability based analysis of the crosswind stability of railway vehicles. Journal of Wind Engineering and Industrial Aerodynamics 95, 493-509 (2007)

Li, X.B., Yang, Z., Zhang, W.H.: Aerodynamics properties of high-speed train in strong wind. Journal of Traffic and Transportation Engineering 9(2), 66-73 (2009)

Xi, Y.H., Mao, J., Li, M.G.: Numerical Study on the Crosswind Effects of High-Speed Train. Journal of Beijing Jiaotong University 34(1), 14-18 (2010)

Zen, Z.S., Xu, Y.G., Wang, L.L.: Study on the running safety of high-speed trains under strong cross winds. Journal of the China Railway Society 28(6), 46-50 (2006)

Gao, G.J., Miao, X.J.: Aerodynamic performance of passenger train on different height of bridge of Qinghai-Tibet railway line under strong cross wind. Journal of Central South University 41(1), 376-380 (2010)

Jiang, C.W., Chen, Z., Guan, X.M.: Numerical Study on Crosswind Loads of Container Trains in Different Length on Plain. Journal of the China Railway Society 32(5), 18-24 (2010)

Khier, W., Breuer, M., Durst, F.: Flow structure around trains under side wind conditions: a numerical study. Computers \& Fluids 29, 179-195 (2000)

Wang, F.J.: Analysis of Computational Fluid Dynamics, Tsinghua, Beijing (2004)

Krajnovic, Siniša: Optimization of aerodynamic properties of high-speed trains with CFD and response surface models. LNACM, vol. 41, pp. 197-211 (2009) 